\title{
Search for signatures of sterile neutrinos with Double Chooz
}

T. Abrahão ${ }^{4,5}$, H. Almazan ${ }^{15}$, J. C. dos Anjos ${ }^{5}$, S. Appel ${ }^{22}$, J. C. Barriere ${ }^{11}$, I. Bekman B. T. J. C. Bezerra $^{18,27}$, L. Bezrukov ${ }^{10}$, E. Blucher ${ }^{7}$, T. Brugière ${ }^{17}$, C. Buck ${ }^{15}$, J. Busenitz ${ }^{2}$, A. Cabrera ${ }^{4,26,28}$, M. Cerrada ${ }^{8}$, E. Chauveau ${ }^{6}$, P. Chimenti ${ }^{5,29}$, O. Corpace ${ }^{11}$, J. V. Dawson ${ }^{4}$, Z. Djurcic ${ }^{3}$, A. Etenko ${ }^{14}$, H. Furuta ${ }^{19}$, I. Gil-Botella ${ }^{8}$, A. Givaudan ${ }^{4}$, H. Gomez ${ }^{4,11}$, L. F. G. Gonzalez ${ }^{24}$, M. C. Goodman ${ }^{3}$, T. Hara ${ }^{13}$, J. Haser ${ }^{15}$, D. Hellwig ${ }^{1}$, L. Heuermann ${ }^{1}$, A. Hourlier ${ }^{4,30}$, M. Ishitsuka ${ }^{20,31}$, J. Jochum ${ }^{23}$, C. Jollet ${ }^{6}$, K. Kale ${ }^{6,17}$, M. Kaneda ${ }^{20}$, M. Karakac ${ }^{4}$, T. Kawasaki ${ }^{12}$, E. Kemp ${ }^{24}$, H. de Kerret ${ }^{4}$, D. Kryn ${ }^{4}$, M. Kuze ${ }^{20}$, T. Lachenmaier ${ }^{23}$, C. E. Lane ${ }^{9}$, T. Lasserre ${ }^{4,11}$, C. Lastoria ${ }^{8}$, D. Lhuillier ${ }^{11}$, H. P. Lima Jr ${ }^{5}$, M. Lindner ${ }^{15}$, J. M. López-Casta no ${ }^{8,13}$, J. M. LoSecco ${ }^{16}$, B. Lubsandorzhiev ${ }^{10}$, J. Maeda ${ }^{13,21}$, C. Mariani $^{25}$, J. Maricic ${ }^{9,32}$, J. Martino ${ }^{18}$, T. Matsubara ${ }^{21,33}$, G. Mention ${ }^{11}$, A. Meregaglia ${ }^{6}$, T. Miletic $^{9,34}$, R. Milincic ${ }^{9,32}$, A. Minotti ${ }^{11,35}$, D. Navas-Nicolás ${ }^{4,8,28}$, P. Novella ${ }^{8,36}$, L. Oberauer ${ }^{22}$, M. Obolensky $^{4}$, A. Onillon ${ }^{11}$, A. Oralbaev ${ }^{14}$, C. Palomares ${ }^{8}$, I. M. Pepe ${ }^{5}$, G. Pronost ${ }^{18,37}$, J. Reichenbacher ${ }^{2,38}$, B. Reinhold ${ }^{15,32}$, S. Schönert $^{22}$, S. Schoppmann ${ }^{15,40,41}$, L. Scola ${ }^{11}$, R. Sharankova ${ }^{20}$, V. Sibille ${ }^{11,30}$, V. Sinev ${ }^{10}$, M. Skorokhvatov ${ }^{14}$, P. Soldin ${ }^{1}$, A. Stahl ${ }^{1}$, I. Stancu ${ }^{2}$, L. F. F. Stokes ${ }^{23}$, F. Suekane ${ }^{4,19}$, S. Sukhotin ${ }^{14}$, T. Sumiyoshi ${ }^{21}$, Y. Sun ${ }^{2,33}$,

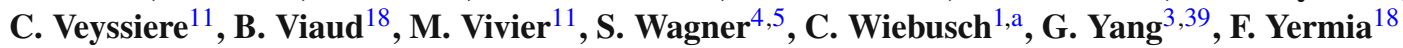

${ }^{1}$ III. Physikalisches Institut, RWTH Aachen University, 52056 Aachen, Germany

2 Department of Physics and Astronomy, University of Alabama, Tuscaloosa, AL 35487, USA

${ }^{3}$ Argonne National Laboratory, Argonne, IL 60439, USA

${ }^{4}$ APC Université de Paris CNRS Astroparticule et Cosmologie, 75006 Paris, France

${ }^{5}$ Centro Brasileiro de Pesquisas Físicas, Rio de Janeiro, RJ 22290-180, Brazil

${ }^{6}$ Université de Bordeaux CNRS/IN2P3 CENBG, 33175 Gradignan, France

${ }^{7}$ The Enrico Fermi Institute, The University of Chicago, Chicago, IL 60637, USA

${ }^{8}$ Centro de Investigaciones Energéticas Medioambientales y Tecnológicas CIEMAT, 28040 Madrid, Spain

${ }^{9}$ Department of Physics, Drexel University, Philadelphia, PA 19104, USA

${ }^{10}$ Institute of Nuclear Research of the Russian Academy of Sciences, Moscow 117312, Russia

${ }^{11}$ IRFU CEA Université Paris-Saclay, 91191 Gif-sur-Yvette, France

12 Department of Physics, Kitasato University, Sagamihara 252-0373, Japan

${ }^{13}$ Department of Physics, Kobe University, Kobe 657-8501, Japan

${ }^{14}$ NRC Kurchatov Institute, 123182 Moscow, Russia

15 Max-Planck-Institut für Kernphysik, 69117 Heidelberg, Germany

${ }^{16}$ University of Notre Dame, Notre Dame, IN 46556, USA

17 IPHC CNRS/IN2P3, Université de Strasbourg, 67037 Strasbourg, France

18 SUBATECH CNRS/IN2P3 Université de Nantes IMT-Atlantique, 44307 Nantes, France

${ }^{19}$ Research Center for Neutrino Science, Tohoku University, Sendai 980-8578, Japan

${ }^{20}$ Department of Physics, Tokyo Institute of Technology, Tokyo 152-8551, Japan

${ }^{21}$ Department of Physics, Tokyo Metropolitan University, Tokyo 192-0397, Japan

22 Physik Department, Technische Universität München, 85748 Garching, Germany

${ }^{23}$ Kepler Center for Astro and Particle Physics, Universität Tübingen, 72076 Tübingen, Germany

${ }^{24}$ Universidade Estadual de Campinas-UNICAMP, Campinas, SP 13083-970, Brazil

25 Center for Neutrino Physics Virginia Tech, Blacksburg, VA 24061, USA

${ }^{26}$ LNCA Underground Laboratory IN2P3/CNRS-CEA, Chooz, France

27 Present Address: Department of Physics and Astronomy, University of Sussex, Falmer, Brighton, UK

28 Present Address: IJC Laboratory CNRS/IN2P3, Université Paris-Saclay, Orsay, France

${ }^{29}$ Present Address: Universidade Estadual de Londrina, 86057-970 Londrina, Brazil

${ }^{30}$ Present Address: Massachusetts Institute of Technology, Cambridge, MA 02139, USA

${ }^{31}$ Present Address: Tokyo University of Science Noda, Chiba, Japan

${ }^{32}$ Present Address: Physics and Astronomy Department, University of Hawaii at Manoa, Honolulu, HI, USA

33 Present Address: High Energy Accelerator Research Organization (KEK), Tsukuba, Ibaraki, Japan

${ }^{34}$ Present Address: Physics Department, Arcadia University, Glenside, PA 19038, USA

${ }^{35}$ Present Address: LAPP CNRS/IN2P3, 74940 Annecy-le-Vieux, France

36 Present Address: Instituto de Física Corpuscular IFIC (CSIC/UV), 46980 Paterna, Spain

${ }^{37}$ Present Address: Kamioka Observatory ICRR, University of Tokyo, Kamioka, Gifu 506-1205, Japan

38 Present Address: South Dakota School of Mines and Technology, 501 E. Saint Joseph St., Rapid City, SD 57701, USA 
${ }^{39}$ Present Address: State University of New York at Stony Brook, Stony Brook, NY 11755, USA

40 Present Address: University of California Department of Physics, Berkeley, CA 94720-7300, USA

${ }^{41}$ Present Address: Lawrence Berkeley National Laboratory, Berkeley, CA 94720-8153, USA

Received: 11 September 2020 / Accepted: 13 July 2021 / Published online: 29 August 2021

(c) The Author(s) 2021

Abstract We present a search for signatures of neutrino mixing of electron anti-neutrinos with additional hypothetical sterile neutrino flavors using the Double Chooz experiment. The search is based on data from 5 years of operation of Double Chooz, including 2 years in the two-detector configuration. The analysis is based on a profile likelihood, i.e. comparing the data to the model prediction of disappearance in a data-to-data comparison of the two respective detectors. The analysis is optimized for a model of three active and one sterile neutrino. It is sensitive in the typical mass range $5 \times 10^{-3} \mathrm{eV}^{2} \lesssim \Delta m_{41}^{2} \lesssim 3 \times 10^{-1} \mathrm{eV}^{2}$ for mixing angles down to $\sin ^{2} 2 \theta_{14} \gtrsim 0.02$. No significant disappearance additionally to the conventional disappearance related to $\theta_{13}$ is observed and correspondingly exclusion bounds on the sterile mixing parameter $\theta_{14}$ as a function of $\Delta m_{41}^{2}$ are obtained.

\section{Introduction}

The standard model of particle physics includes three flavors of neutrinos that interact through the weak force with other particles [53]. The neutrino flavors are identified by the corresponding charged lepton in charged current interactions. With the discovery $[16,36]$ of neutrino oscillations $[46,49]$, it became clear that neutrinos have mass. Currently the majority of observations is consistent with the standard picture of three mass eigenstates $\left(v_{1}, v_{2}, v_{3}\right)$ mixing with the flavor eigenstates $\left(v_{e}, v_{\mu}, v_{\tau}\right)$. The mixing is described by a $3 \times 3$ unitary matrix (PNMS matrix), parametrized by three mixing angles $\theta_{12}, \theta_{13}, \theta_{23}$ as well as a CP violating phase $\delta$ and two Majorana phases if neutrinos are Majorana particles.

The neutrino experiments Double Chooz, Daya Bay, and RENO contributed to the field by establishing the third oscillation mode that is related to the mixing angle $\theta_{13}[3,17,21]$. These experiments observe the disappearance of $\bar{v}_{e}$ from nuclear reactors by measuring the flux at different distances. The concept of multiple identical detectors has proven crucial in controlling and reducing systematic uncertainties. Today, the oscillation angle $\theta_{13}$ is the most precisely measured oscillation parameter [53].

There have been speculations about the existence of additional neutrinos that are non-interacting with matter, see e.g.

H. de Kerret: Deceased.

a e-mail: wiebusch@physik.rwth-aachen.de
[1]. These thoughts are supported by experimental anomalies reported by the LSND [14] and MiniBooNE [15] neutrinobeam experiments as well as the so-called reactor [47] and gallium $[2,9,39]$ anomalies, where the observed $\bar{v}_{e}$ and $v_{e}$ fluxes are roughly 5-10\% less than the theoretical predictions. However, the uncertainty of those predictions remains an open question and our latest results [41] indicate a possible underestimation of the reactor flux prediction. Though this deficit is marginally compatible with the uncertainty of the flux prediction, it could be also interpreted as disappearance due to oscillation with additional neutrino states. Recently, the Neutrino-4 collaboration has reported [52] indications of a spectral distortion at short baseline to the reactor that would be consistent with the oscillation hypothesis. This result is subject of ongoing discussions [24,31,51]. Particularly it has been reviewed in [28] considering the validity of the Wilkstheorem, thus resulting in a reduced significance. Note that in this paper we report a very similar effect of reduced significance with respect to Wilks' theorem in our measurement. From a phenomenological perspective it is important to emphasize that consistency of all today's global data within a single simple solution remains an unsettled open debate, see e.g. [33].

The simplest extension of the standard oscillation picture is a $3+1$ model [1]. Though this model cannot consistently explain all experimental anomalies, its few parameters make it well suited as a benchmark model in the following discussions. Here, one additional sterile, i.e. not weakly interacting, neutrino mixes with the three active neutrino states. This results in an additional mass state $m_{4}$ and an extension of the mixing matrix to $4 \times 4$ with the additional parameters $\theta_{14}$, $\theta_{24}, \theta_{34}$, and additional $\mathrm{CP}$ violating phases.

In this picture, a non-zero mixing of reactor $\bar{v}_{e}$ with a sterile neutrino will result in a disappearance, superimposed to the standard oscillation related to $\theta_{13}$. Assuming small mixing and baselines relevant for the Double Chooz experiment, only the parameters $\theta_{14}$ and the difference of squared masses $\Delta m_{41}^{2} \equiv m_{4}^{2}-m_{1}^{2}$ are relevant [43], and the survival probability of $\bar{v}_{e}$ as a function of distance $L$ and energy $E$ can be approximated by

$$
\begin{aligned}
& P_{\bar{v}_{e} \rightarrow \bar{v}_{e}}(E, L) \approx 1-\sin ^{2}\left(2 \theta_{13}\right) \sin ^{2}\left(1.267 \frac{M e V}{e V^{2} m} \cdot \frac{\Delta m_{e e}^{2} L}{E}\right) \\
& -\sin ^{2}\left(2 \theta_{14}\right) \sin ^{2}\left(1.267 \frac{M e V}{e V^{2} m} \cdot \frac{\Delta m_{41}^{2} L}{E}\right)
\end{aligned}
$$



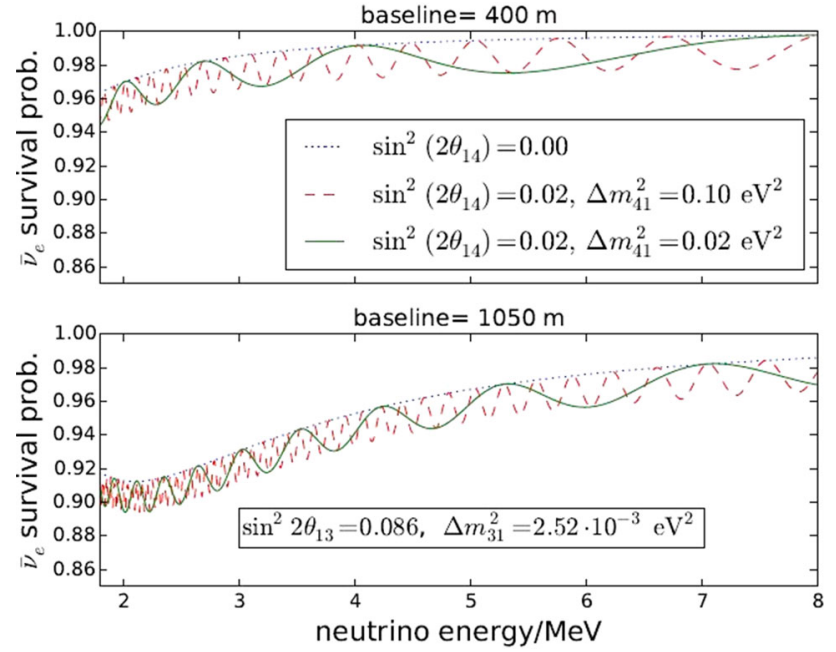

Fig. 1 Survival probability of reactor $\bar{v}_{e}$ as a function of the energy for the baselines of the ND (top) and FD (bottom) for different benchmark oscillation parameters $\theta_{14}$ and $\Delta m_{41}^{2}$. The dotted line corresponds to the no-sterile case, where the survival probability is governed by the conventional $\theta_{13}$ oscillation. The dashed and solid lines show two different examples of sterile mixing

The first sine term corresponds to the disappearance related to the standard $\theta_{13}$ mixing while the second sine term describes the additional disappearance due to the mixing with the sterile neutrino state. The term $\Delta m_{e e}^{2}$ is a shorthand for $\cos ^{2} \theta_{12} \Delta m_{31}^{2}+\sin ^{2} \theta_{12} \Delta m_{32}^{2}$.

The effect is displayed in Fig. 1 for baselines of $400 \mathrm{~m}$ and $1050 \mathrm{~m}$ corresponding to the average distances of the nuclear reactors to the two Double Chooz detectors. The existence of sterile neutrinos with non-zero mixing leads to the additional disappearance superimposed on the conventional oscillation. The amplitude of this oscillation is given by the parameter $\sin ^{2}\left(2 \theta_{14}\right)$. The oscillation frequency seen in the energy-dependence is proportional to the difference of squared masses. For mass differences of $\Delta m_{41}^{2} \gg 0.1 \mathrm{eV}^{2}$, oscillations become fast. Given the experimental energy resolution, they become eventually indistinguishable from a global normalization change. Similarly, for small mass-square differences $\Delta m_{41}^{2} \approx \Delta m_{e e}^{2} \simeq 2.5 \times 10^{-3} \mathrm{eV}^{2}$ the disappearance becomes indistinguishable from the conventional oscillation with $\theta_{13}$. Note, that the above approximation is only used for illustrative purposes and for all numerical calculations in this analysis we use the full four-flavor propagation code nuCraft [55].

The position of the two Double Chooz detectors has been optimized for the measurement of $\theta_{13}$ assuming $\Delta m^{2} \approx$ $2.5 \times 10^{-3} \mathrm{eV}^{2}$. For an energy range of detected reactor neutrinos between about from 1 to $8 \mathrm{MeV}$ and the two baselines of $400 \mathrm{~m}$ and $1050 \mathrm{~m}$, the probed $L / E$ range for the disappearance of $\bar{v}_{e}$ is approximately $50-1000 \mathrm{~m} / \mathrm{MeV}$. For larger mass differences, shorter baselines are desirable in

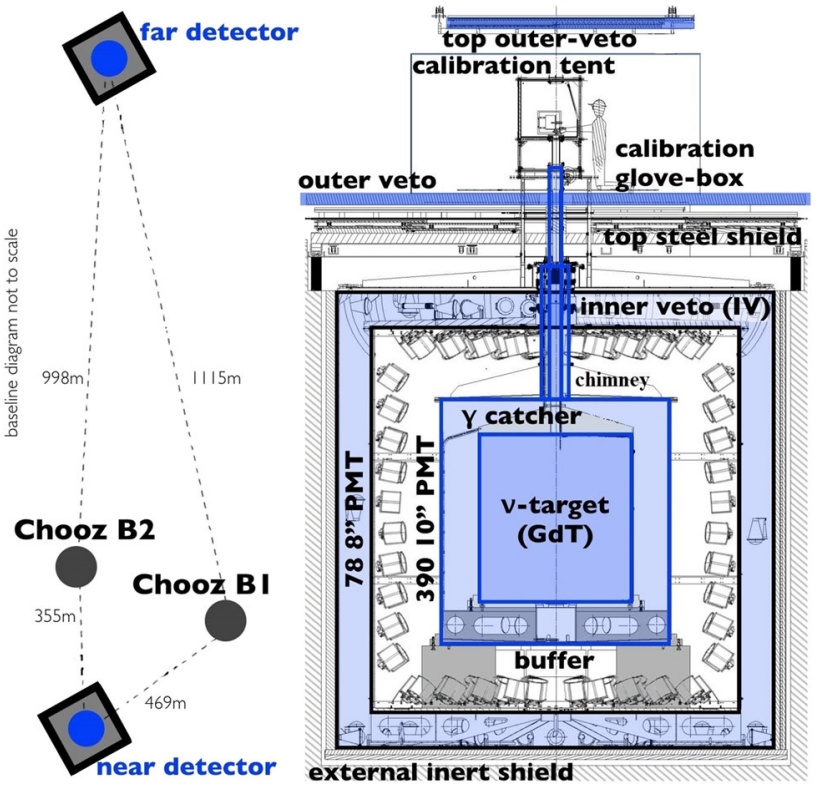

Fig. 2 The Double Chooz experiment. Left: arrangement of the two detectors far and near with respect to the nuclear reactors. Right: design of a Double Chooz detector. Figure modified from [41]

order to observe the un-oscillated flux with a near detector. This is realized by short-baseline experiments, Bugey-3 [32] and more recently DANSS [18], NEOS [42], Neutrino4 [52], PROSPECT [26], SoLID [8], and STEREO [19,20], that target mass-square differences on the $\mathrm{eV}^{2}$ scale. The probed $L / E$ range for these experiments is typically $1-20$ $\mathrm{m} / \mathrm{MeV}$. Therefore the here presented search is complementary in probed $L / E$ as well as lower probed mass-square differences below $0.1 \mathrm{eV}^{2}$; see also [34].

\section{Experimental setup}

The Double Chooz experiment consists of two nearly identical gadolinium-doped liquid scintillator detectors [25] located close to the Chooz-B nuclear power plant, see Fig. 2. The power plant consists of two nuclear reactors of type N4, $165 \mathrm{~m}$ apart with a thermal power of about $4.25 \mathrm{GW}$ each. The far (near) detector is located underground with an overburden of about $300 \mathrm{~m}(120 \mathrm{~m})$ water equivalent at a distance of $1115 \mathrm{~m}$ and $998 \mathrm{~m}$ ( $469 \mathrm{~m}$ and $355 \mathrm{~m}$ ) to the reactor cores.

Details of the detectors are described in $[3,4,6,41]$. The detectors are constructed in an onion-like structure with a central detector made of four concentric cylindrical tanks. The innermost acrylic vessel contains $10.3 \mathrm{~m}^{3}$ gadolinium loaded liquid scintillator called the $v$-target. The $v$-target is surrounded by the $\gamma$-catcher, filled with $22.5 \mathrm{~m}^{3}$ liquid scintillator without gadolinium loading. Both central volumes serve as the neutrino target. A neutrino interacting in the target by inverse beta decay $\left(\bar{v}_{e}+p \rightarrow e^{+}+n\right)$ [54] pro- 
duces the characteristic signature of a delayed coincidence well known since the early days of neutrino experiments [29]. This is formed by a prompt signal from the positron and its annihilation and then the delayed signal from the capture of the thermalized neutron by either gadolinium or hydrogen. Though increasing the rate of accidental background events, the use of both types of captures in a combined data set greatly enlarges the sensitive volume and thus the statistics of detected neutrinos as well as reducing some of the systematic uncertainties. This technique, called total neutron capture, has been developed by the Double Chooz experiment for the most recent $\theta_{13}$ analysis [41] and is applied also for this analysis.

The central target volumes are surrounded by a buffer volume filled with mineral oil, shielding the inner volume from radioactivity, partly from 390 10-inch PMTs that are installed on the inner wall of the stainless steel buffer tank and observe the target. Optically separated from these inner volumes is the inner veto. That is a $50 \mathrm{~cm}$ thick cylindrical volume filled with liquid scintillator and equipped with 78 8 -inch PMTs. It actively shields the inner detector by tagging cosmic-ray induced muons, gammas, and neutrons from outside the detector. Shields of $15 \mathrm{~cm}$ thick demagnetized steel ( $1 \mathrm{~m}$ water) surround the inner veto of the far (near) detector, suppressing external gamma rays. A chimney in the top center allows deploying radioactive sources for calibration. Above the detector is the outer veto detector that adds to the shielding and allows for evaluating the efficiency of the inner veto detector.

Several key aspects of the Double Chooz experiment are important to this analysis. A main goal is avoiding dependencies on absolute predictions of the neutrino flux from the reactors as well as detection efficiencies. Therefore we perform a direct comparison of the event rates measured in the two identical detectors, in the following referred to as datato-data approach. This results in the cancellation of most reactor flux related uncertainties as well as detection efficiencies and some of the background uncertainties in the measurement of $\bar{v}_{e}$ disappearance. Furthermore, due to the presence of only two, relatively close reactor cores, the geometry constitutes well defined baselines from the reactors to the detectors which is important for testing faster oscillation modes than the $\theta_{13}$ oscillation (see Fig. 1). The two detectors are situated close to the so-called iso-flux line, where the ratio of neutrino fluxes from the two reactors is the same for both detectors, i.e. the relative contribution from the two reactors is very similar in the two detectors, further reducing the reactor uncertainty. Another important aspect is that we include measurements when one of the reactors or even both reactors were switched off. These data allow for directly measuring the backgrounds and their spectral properties $[5,40]$. In this analysis, the data from these off-reactor phases are used to construct templates of the energy distribution of backgrounds as well as the uncertainties of these templates for the fit to data. Additionally, the total rate is used to constrain the background rates.

Experimental backgrounds include uncorrelated backgrounds, where a single event appears in a random coincidence with another event, as well as correlated backgrounds that mimic both the prompt and the delayed event. The dominant sources of uncorrelated backgrounds are natural radioactivity and instrumental noise such as spontaneous light emission in the PMT bases of the far detector [7]. Correlated backgrounds are mostly caused by secondary products from cosmic ray air induced atmospheric muons that pass close or through the detectors. Muons reaching the detector are detected with high efficiency and cause an active veto of $1.25 \mathrm{~ms}$ duration. However, background events arise by (i) fast neutrons from interactions in the rock close to the detector entering the neutrino target, (ii) long lived isotopes, in particular ${ }^{9} \mathrm{Li}$ [40], that undergo $\beta$-decays followed by neutron emission, and (iii) low energy stopping muons that enter the detector through the chimney and decay by emission of a Michel electron. All these backgrounds are considerably reduced during the data selection and the remainder are measured with specific methods and in dedicated campaigns, e.g. during reactor-off phases.

The data of this analysis are identical to the selection described in [41] and are separated into three data sets. The first (FD-I) has been collected with the far detector prior to commissioning of the near detector and consists of 455.21 days of dead and down-time corrected livetime, collected between April 2011 and January 2013. The second set (FDII) has been collected with the far detector during operation of both detectors and consists of 362.97 days of livetime collected between January 2015 and April 2016. The third set (ND) are the data collected during the same period with the near detector and corresponds to 257.96 days of livetime. Note that the effective livetime of the ND data is reduced with respect to the FD-II data, because the larger muon rate in the near detector causes a larger dead-time due to vetoing. While the previously described data has been collected during operation of at least one reactor, additionally 7.16 days of livetime with both reactors switched off during the FDI phase are used to determine the total rate of background events.

\section{Analysis method}

The analysis is based on a profile likelihood ratio (see e.g. Cowan in [53]) that has already been exploited by Double Chooz for a measurement of $\theta_{13}$ in [50] and has also been used internally to confirm the result in [41]. The test statistic is defined as the ratio of maximum likelihoods for tested model parameters $\boldsymbol{\eta}=\left\{\sin ^{2} 2 \theta_{14}, \Delta m_{41}^{2}\right\}$ with respect to the glob- 
ally largest likelihood value which is found for the parameters $\hat{\boldsymbol{\eta}}=\left\{\sin ^{2} \hat{2} \theta_{14}, \Delta \hat{m}_{41}^{2}\right\}$. This defines the test statistic for the given data set $\mathbf{x}$ and model parameters $\eta$

$\lambda(\mathbf{x}, \boldsymbol{\eta})=-2 \cdot \ln \frac{\sup \mathcal{L}(\mathbf{x} \mid \boldsymbol{\eta}, \boldsymbol{\xi})}{\sup \mathcal{L}(\mathbf{x} \mid \hat{\boldsymbol{\eta}}, \hat{\boldsymbol{\xi}})}=-2 \Delta \ln (\mathcal{L})$

In addition to the two model parameters $\eta$ that describe a sterile neutrino signal, the reactor fluxes, detector responses, systematic uncertainties and backgrounds are modeled by a total number of 298 additional and partly correlated parameters $\boldsymbol{\xi}$ (see below for details). These parameters are treated as nuisance parameters in the fit. They are optimized separately for each respective signal hypothesis with $\boldsymbol{\xi}$ representing those nuisance parameters that maximize the local likelihood for the tested $\eta$.

For the test of a potential oscillation signal from sterile neutrinos, we compare the best-fit standard 3-flavor model (null hypothesis, $\eta_{0}$ ), described by the two parameters $\sin ^{2} 2 \theta_{14}=0$ and $\Delta m_{41}^{2}=0$, to the globally best fit $3+1$ sterile neutrino model (signal hypothesis) for the parameters $\hat{\eta}$ that maximize the likelihood of the data $\mathbf{x}$. Note that specifically the null hypothesis $\eta_{0}$ is degenerate with respect to the two parameters $\eta$ because only one of them fixed to zero is sufficient to model a no-oscillation signal. Furthermore, $\boldsymbol{\eta}_{0}$ is a special case, nested within the parameter space of the signal hypothesis resulting in $\lambda\left(\mathbf{x}, \eta_{0}\right) \geq 0$.

The likelihood itself is implemented as a product of multiplicative terms with the Poissonian likelihoods $\mathcal{P}\left(n_{i}, \mu_{i}\right)$ of the observed number of events $n_{i}$ in the energy bin $i$ in all three data sets $d \in\{N D, F D-I, F D-I I\}$ multiplied with Gaussian prior functions $\mathcal{G}$ on external nuisance parameters

$$
\begin{aligned}
\mathcal{L}(\mathbf{x} \mid \boldsymbol{\eta}, \boldsymbol{\xi})= & \prod_{d \in\{N D, F D-I, F D-I I\}} \prod_{i \in\left[E_{\text {min } \left.\ldots E_{\text {max }}\right]}\right.} \\
& \mathcal{P}\left(n_{d, i}, \mu_{d, i}(\boldsymbol{\eta}, \boldsymbol{\xi})\right) \\
& \cdot \mathcal{P}\left(n_{o f f}, \mu_{o f f}(\boldsymbol{\xi})\right) \\
& \cdot \prod_{a \in \xi}\left(\mathcal{G}\left(a, a_{0}, \sigma_{a}\right)\right) \\
& \cdot \prod_{\mathbf{b} \in \boldsymbol{\xi}}\left(\mathcal{G}\left(\left(\mathbf{b}-\mathbf{b}_{0}\right)^{T} \mathbf{V}_{b}^{-1}\left(\mathbf{b}-\mathbf{b}_{0}\right)\right)\right)
\end{aligned}
$$

Here $\mu_{d, i}(\boldsymbol{\eta}, \boldsymbol{\xi})$ denotes the summed bin expectations of signal and backgrounds as a function of the model parameters. The second term is the Poisson probability of the observed event number during the reactor-off phases for the background expectation as a function of the nuisance parameters. The third term describes Gaussian priors for all single, uncorrelated nuisance parameters $a$ with the expectation $a_{0}$ and the uncertainty $\sigma_{a}$. The fourth term describes Gaussian priors for all nuisance parameters $\mathbf{b}$ that are correlated, described by the expectation $\mathbf{b}_{0}$ and the covariance matrix $\mathbf{V}_{b}$.
The data are binned for each of the three sets in 38 bins between 1 to $20 \mathrm{MeV}$ with custom bin sizes. The region up to $8 \mathrm{MeV}$ which is dominated by measured reactor $\bar{v}_{e}$ has 28 bins of $0.25 \mathrm{MeV}$ size. Above $8 \mathrm{MeV}$, bins are background dominated but are included in the fit as they allow for constraining the background rates. Due to the lower statistics, larger bin sizes are used. These are 4 bins of $0.5 \mathrm{MeV}$ size between 8 to $10 \mathrm{MeV}$, where rare isotopes $\left({ }^{9} \mathrm{Li}\right)$ dominate and 4 bins of $2 \mathrm{MeV}$ size between 12 to $20 \mathrm{MeV}$, where fast neutrons dominate. In the intermediate region $10-12 \mathrm{MeV}, 2$ bins of $1 \mathrm{MeV}$ size are used.

Systematic uncertainties are modeled by the following nuisance parameters $\xi$ in the analysis (more details are given in [37]):

- The normalizations of the reactor flux expectation for each energy bin are free fit parameters. This approach is independent of existing reactor flux predictions and the normalizations are only constrained by the data-to-data comparison of rate and shape of the data in each detector. This way, known discrepancies of reactor flux models $[23,41,42,44]$, being independent of the baseline, do not bias the fit, however, at the price of a slightly reduced sensitivity. The basis of the above approach is a large correlation in the observed reactor flux for the three data sets FD-I, FD-II, ND. Because of different running times, this assumption is only approximate, $(99.75 \%$ for FD-II and ND, 93.20\% for FD-I and FD2, 93.10\% for FD-I and ND). Therefore, we model additional constraints on the normalization of each energy bin of the three data sets with a total of $3 \times 41$ reactor flux parameters between 1 to $11.25 \mathrm{MeV}$. The number of parameters is determined by the greatest common divisor of the bin widths to create a uniform binning. These bins form the basis of an area conserving spline, which is energy corrected and later integrated over in the original binning. These parameters are correlated between the data sets with the above correlation factors and additionally we allow for uncorrelated shape deviations with a $41 \times 41$ covariance matrix for each data set, that is determined from the reactor flux prediction.

- The conventional oscillation parameters $\sin ^{2} 2 \theta_{13}$ and $\Delta m_{e e}^{2}$ are free parameters. While $\Delta m_{e e}^{2}$ is seeded with the global best value from [48] and is constrained with a prior corresponding to its uncertainty, $\sin ^{2} 2 \theta_{13}$ is left unconstrained. The latter ensures that assumptions about the value, which has itself been largely determined in reactor neutrino experiments, cannot introduce a bias. By this, $\sin ^{2} 2 \theta_{13}$ can acquire a different best-fit value for $\theta_{14} \neq 0$.

- Backgrounds are modeled with free parameters for rate and shape. The shape of the contribution from rare isotopes $\left({ }^{9} \mathrm{Li}\right)$ is assumed identical between the three data 
sets. It has been determined experimentally by a dedicated selection of events correlated in time and space with tagged muon events [6] and it is modeled with 38 shape parameters. The rate is assumed identical for FD-I and FD-II but is different for ND. Both total rates are not constrained by a prior but are determined by the data as free parameters during the fit.

The rates and shapes of accidental backgrounds have been determined by time-scrambled experimental data for each data set and are individually modeled by 38 parameters for the shape and one parameter for the rate. These parameters are assumed uncorrelated for the three data sets, to account for changes in data taking over time and differences in the detectors but are constrained with a prior that reflects the uncertainty in the determination of the rates.

The fast neutron and stopping muon backgrounds are modeled as $R(E)=R_{0}\left(a \cdot E+b \cdot \exp (-\lambda \cdot E)\right.$. Here, $R_{0}$ is the total rate and the three shape parameters $\lambda, a$, and $b$ are further constrained by the normalization. The shapes are assumed to be fully correlated between the data sets, while the rates are the same for for FD-I and FD-II but independent for ND.

A special case is the small constant rate of $\bar{v}_{e}$ from the reactor fuel that has been determined during FD-I reactor-off phases to $0.58 \pm 0.17 d^{-1}$. As these neutrinos undergo the same oscillation, this is modeled in the fit with the nominal oscillated shape expectation for $\bar{v}_{e}$ and the rate is constrained by a prior corresponding to this reactor-off rate.

- The uncertainties in the detector response are modelled identically to [41] by second order polynomials. They take into account the non-linearity of the visible energy response of the scintillator, the non-uniformity within the detector, and the charge non-linearity of the photomultiplier and electronics response. After analyzing the correlations of these effects where we assume the energy response of the scintillator to be fully correlated but the other effects to be uncorrelated between the data-sets, the 9 polynomial coefficients can be expressed by 7 independent parameters. In addition to the energy response, the total detection efficiency is subject to uncertainty, dominated by the uncertainty of the total target mass. This is modeled by a total of three constrained and partly correlated parameters.

The resulting expectations of reactor $\bar{v}_{e}$ as well as the backgrounds for the default model are shown in Fig. 3 in comparison to the experimental data for all three data sets. In addition to the above parameters we have tested additional uncertainties but their effect was found to be negligible. In particular it was shown that the choice of mass ordering has no relevant impact on the analysis.
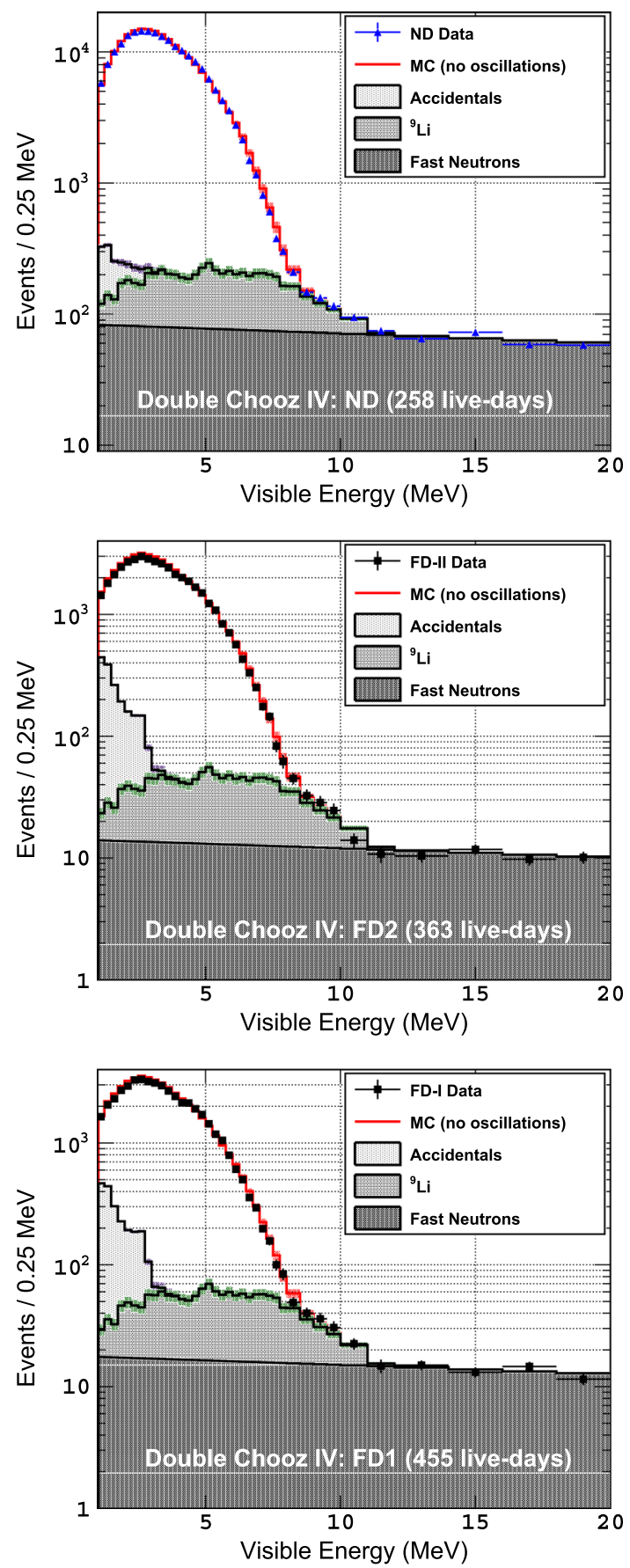

Fig. 3 Visible energy distributions of the prompt events in the final data set. The ND (top) data is plotted with blue triangles and the FDII (middle) and FD-I data are displayed as black squares. The different background model contributions are shown as stacked histograms where green indicates the long-lived isotopes (lithium) background, blue the accidental background and gray the fast neutron and stopping muon background. The red line indicates the total prediction from reactor models assuming no oscillations including the backgrounds 
The above fit has been extensively tested. These tests include a detailed validation of the $\theta_{13}$ fit in the absence of a sterile signal that was found in good agreement to the published standard analyses of Double Chooz. Here, the relative impact of each systematic uncertainty has been evaluated by performing fits excluding the corresponding nuisance parameter $(\mathrm{N}-1)$ or fits including exclusively this parameter on top of statistical uncertainties (stat +1$)$. All resulting uncertainties have been found in good agreement with the standard analysis [41].

For the validation of the detection of a sterile signal, studies of pseudo experiments with injected signal and blind datachallenges have been performed. Furthermore, the impact of each systematic parameter and other experimental effects, such as the spectral distortion at $5 \mathrm{MeV}$ have been tested. Here, it was verified that the fit results in an unbiased estimation of the parameters $\sin ^{2} 2 \theta_{14}$ and $\Delta m_{41}^{2}$.

\section{Test statistic}

The maximum likelihood is numerically obtained by minimizing the negative $\log (\mathcal{L})$. However, finding the global minimum and $\hat{\eta}$ is numerically challenging because the fit does not converge for arbitrary combinations of initial signal and nuisance parameters to the global minimum. Therefore, the full phase space of signal parameters $\eta$ is scanned by performing a numerical fit of the parameters $\xi$ for each scan point. The result of such a scan is shown in Fig. 4 for an Asimov data set [30] based on Monte Carlo simulations of the null hypothesis of only standard oscillations. As the Asimov data set represents the mean expectation for this hypothesis, we thus find $\lambda(\mathbf{x})=0$ for $\sin ^{2}\left(2 \theta_{14}\right)=0$ corresponding to the injected null hypothesis.

As noted above, the null hypothesis is a special case nested within the more general signal hypothesis. The test statistic thus allows for a hypothesis test for a sterile signal i.e. nonzero $\boldsymbol{\eta}$ with respect to the no-sterile case $\boldsymbol{\eta}_{0}=\mathbf{0}$ based on the likelihood ratio. If applied, Wilks' theorem [56] would predict that the test statistic $T S=\lambda\left(\mathbf{x}, \boldsymbol{\eta}_{0}\right)$ follows a $\chi^{2}$ distribution with two degrees of freedom corresponding to the difference in degrees of freedom of the signal and null hypotheses. However, the preconditions for Wilks' theorem are not fulfilled. First, the two parameters $\sin ^{2} 2 \theta_{14}$ and $\Delta m_{41}^{2}$ are degenerate in case of the null hypothesis. Any combination of these with one of the two parameters equal to zero is sufficient for fulfilling the null hypothesis even if the other parameter has a non-zero value. In many practical applications one can accommodate the problem by introducing an effective degree of freedom $1 \leq n_{\text {eff }} \leq 2$ and the value of $n_{\text {eff }}$ can be estimated by pseudo experiments with the method introduced by Feldman and Cousins [35]. Secondly, the expectation value of partial derivatives with respect to

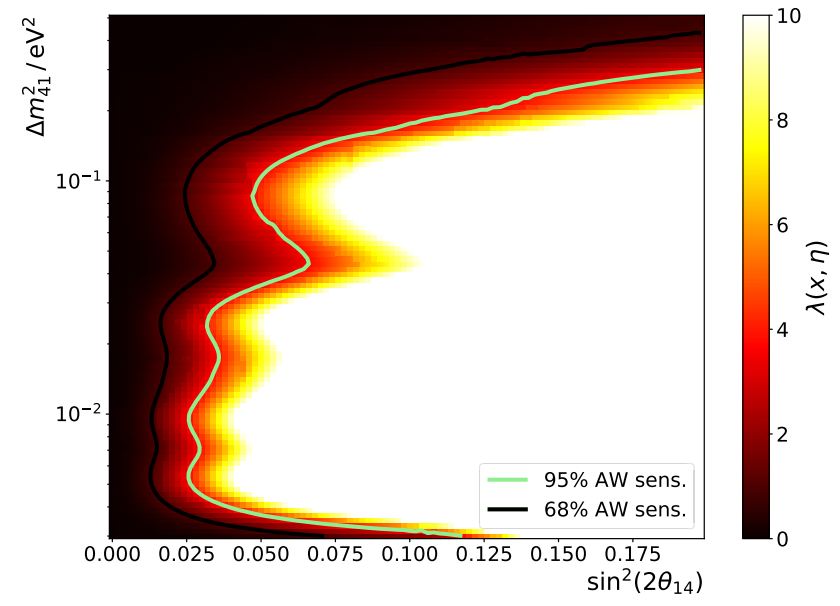

Fig. 4 Test statistic $\lambda(\mathbf{x}, \boldsymbol{\eta})$ for an Asimov data set $\mathbf{x}$ of the null hypothesis for a scan of the signal parameter space. All values of $\sin ^{2} 2 \theta_{14}=0$ represent the null hypothesis of no-sterile oscillations and correspondingly $\lambda=0$ for the Asimov data set. The color scale is clipped at $\lambda=10$. The lines represent the $68 \%$ and $95 \%$ sensitivity (see text) for constraining $\sin ^{2} 2 \theta_{14}$ as a function of $\Delta m_{41}^{2}$

the parameters $\left\|\left\langle\frac{\partial^{2} \mathcal{L}(\mathbf{x} \mid \eta)}{\partial \eta_{i} \partial \eta_{j}}\right\rangle\right\|$ should form a positively definite matrix. Due to the oscillatory structure of the signal hypothesis, this is not the case here. A data fluctuation in any of the energy bins can be better described by some signal hypotheses that correspond to such an oscillatory pattern in the detectors. As a matter of fact, multiple, very different signal parameters can lead - within the experimental resolutions - to similar patterns. In an illustrative picture, for a statistical fluctuation of the experimental data bins in energy, multiple different combinations of signal parameters allow for a slightly improved description of the data with respect to the null hypothesis. As a result, multiple minima of the test statistic can be found within the signal parameter space. However, the existence of several minima implies that the above matrix of derivatives is zero in some points of the parameter space.

As verification of the above discussion, Fig. 5 shows an example analysis for a pseudo data set that was generated from a Monte Carlo simulation of the null hypothesis. The occurrence of multiple minima of the test statistic is well visible. As apparent features, these minima are horizontally elongated and thus correspond to a fixed value of $\Delta m_{41}^{2}$. Repeated pseudo experiments show similar features with, however, different number of minima and locations in each experiment. This supports the interpretation that for each possible statistical representation of the null hypothesis, multiple signal hypotheses can be found that describe the observed data slightly better than the average expectations from the null hypothesis. Each such solution requires a fixed oscillation length and is usually found close to the sensitivity-line beyond the region where a stronger signal would likely cause a more significant observation. More details on these obser- 


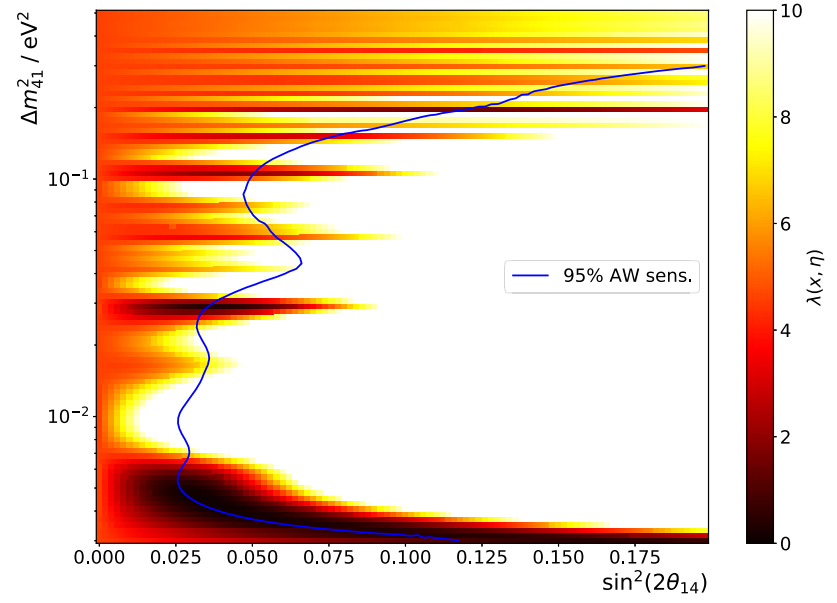

Fig. 5 Example analysis of a pseudo data set representing the null hypothesis. The data set was generated with Poissonian fluctuations from a Monte Carlo data set. The blue line represent the $95 \%$ sensitivity (as defined in the text) for constraining $\sin ^{2} 2 \theta_{14}$ as a function of $\Delta m_{41}^{2}$

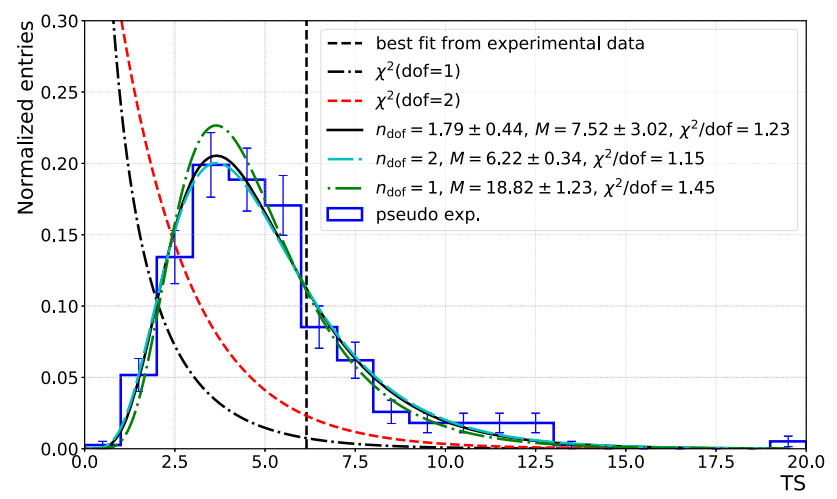

Fig. 6 Expected distribution of test statistic values as obtained from 390 pseudo experiments of the null hypothesis. Also shown is the expectation for a $\chi^{2}$-distribution with one and two degrees of freedom and various modified distribution functions (see text)

vations can be found in [37]. We note that this has been independently discussed in [13] for short-baseline sterile neutrino searches and very recently in [28].

As a consequence, the distribution of the test statistic values $T S=\lambda\left(\mathbf{x}, \boldsymbol{\eta}_{0}\right)$ cannot be approximated by a $\chi^{2}$ distribution but has to be derived from an ensemble study of pseudo experiments. Due to the huge computational effort for scanning the full parameter space, this has been possible only for limited statistics of a few hundred pseudo experiments. The resulting test statistic values $T S$ when comparing the global minimum to the null hypothesis are shown in Fig. 6. It can be clearly seen that the test statistic strongly deviates from $\chi^{2}$-distributions of one and two degrees of freedom. Motivated by the fact, that the choice of the best of several random minima in the parameter space introduces a selection with trials (often called lookelsewhere effect), we introduce a trial factor in three ver-

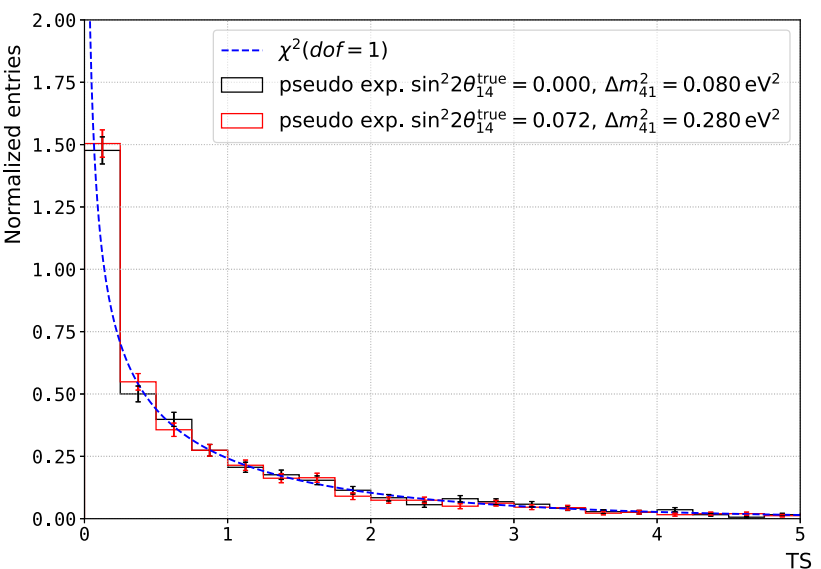

Fig. 7 Test statistic for fixed values of $\Delta m_{41}^{2}$. Shown are the results from 1999 pseudo experiments when fitting $\sin ^{2} 2 \theta_{14}$ for 100 discrete values of $\Delta m_{41}^{2}$ for the injected null hypothesis. For comparison, the expectation from a $\chi^{2}$ distribution of one degree of freedom is shown. Additionally the test statistic for 1997 pseudo experiments of an injected signal is shown. Here the median of the fit $\sin ^{2} 2 \theta_{14}^{\text {med }}$ has been determined for each of the 100 tested $\Delta m_{41}^{2}$ values. The test statistic is then evaluated with $\sin ^{2} 2 \theta_{14}^{\text {med }}$ instead of $\sin ^{2} 2 \theta_{14}=0$ as the null hypothesis. All distributions are found to be consistent

sions of a modified approximation of the test statistic. For this, we calculate the probability distribution $f_{M}(x)$ of the largest $\chi^{2}$ value $x$ from an ensemble $M$ trials. This results to $f_{M}(x)=M \cdot \chi^{2}\left(x, n_{d o f}\right) \cdot\left(\int_{0}^{x} \chi^{2}\left(y, n_{d o f}\right) d y\right)^{M-1}$ where $\chi^{2}\left(x, n_{d o f}\right)$ is the p.d.f. of a single trial. Three versions of this approximation with $M$ as a free parameter are fitted to the pseudo experiments, using $\chi^{2}$ distributions of one, two, and a fitted degree of freedom $n_{d o f}$. All three versions describe the observed test statistic reasonably well. Particularly the case of $n_{d o f}=2$ results in a fitted $M \simeq 6$ which agrees well with the observations in pseudo experiments.

The situation becomes simpler, when taking into account that multiple values of $\Delta m_{41}^{2}$ can cause a minimum in the test statistic. In a modified hypothesis, we can define the sensitivity as the ability to test values of $\sin ^{2} 2 \theta_{14}$ as a function of $\Delta m_{41}^{2}$. When analyzing the pseudo experiments in a raster-scan for distinct fixed values of $\Delta m_{41}^{2}$ and varying only $\sin ^{2} 2 \theta_{14}$ [35], a distribution that is well compatible with the expectation from a $\chi^{2}$ distribution with one degree of freedom is found as shown in Fig. 7. Also for an injected signal, the test statistic with respect to the median expectation of the null hypothesis is consistent and also described by the same $\chi^{2}$ distribution. This is a good confirmation of our assumption that the observed trials are only related to different degenerated oscillation lengths. This test shows that in this case the test statistic can be well described with a $\chi^{2}$-distribution of one degree of freedom in agreement with Wilks' theorem. 


\section{Sensitivity}

We define the sensitivity, in the following denoted as AsimovWilks' (AW) sensitivity, by the boundary value $\sin ^{2} 2 \theta_{14}$ as a function of $\Delta m_{41}^{2}$ where the test statistic of the Asimov data set has a value $\langle\lambda(\mathbf{x})\rangle \geq 3.84$ (or $\langle\lambda(\mathbf{x})\rangle \geq 1)$. This corresponds to the boundary of the median signal expectation where in case of absence of a signal $95 \%$ (or $68 \%$ ) of experiments obtain a smaller value of $\sin ^{2} 2 \theta_{14}$. Note, that because an Asimov data set of the null hypothesis contains no fluctuations, the use of Wilks' theorem is valid and not in contradiction with the above discussion. Furthermore, the best found likelihood always corresponds to the injected null hypothesis. As the null hypothesis is degenerate in $\Delta m_{41}^{2}$, this choice of sensitivity corresponds effectively to a one-dimensional sensitivity on the maximum allowed value of $\sin ^{2} 2 \theta_{14}$ as a function of $\Delta m_{41}^{2}$, also known under the term raster-scan [45]. This choice is consistent with the final choice of experimental limit that will be discussed below. Also, choosing a $\chi^{2}$ distribution of one degree of freedom for the test statistic value of $95 \%$ coverage is a result of this degeneracy.

This choice of sensitivity marks the region, where larger values of $\sin ^{2} 2 \theta_{14}$ are expected to lead to indications of a signal on the level of two (or one) standard deviations but is also closely related to the ability of constraining $\sin ^{2} 2 \theta_{14}$ in the absence of a signal. These sensitivities are shown as lines in Figs. 4 and 5. The statistical coverage of the AW-sensitivity as well as the unbiased estimation of the model parameters $\sin ^{2} 2 \theta_{14}$ and $\Delta m_{41}^{2}$ have been verified with ensembles of pseudo data in [37].

For small values of $\Delta m_{41}^{2} \lesssim 5 \times 10^{-3} \mathrm{eV}^{2}$, the sensitivity becomes weaker as the disappearance becomes ambiguous with conventional oscillations whose energy dependence is given by $\Delta m_{e e}^{2}$. The free nuisance parameter $\sin ^{2} 2 \theta_{13}$ becomes degenerate with $\sin ^{2} 2 \theta_{14}$ and the sensitivity decreases. Also towards large values of $\Delta m_{41}^{2} \gtrsim 0.3 \mathrm{eV}^{2}$ the sensitivity decreases, because oscillations become fast, and the disappearance turns into an overall deficit for both detectors. For the data-data fit approach as implemented here, an oscillation signal would thus become increasingly indistinguishable from an overall change of the reactor flux normalization. We have tested that by additionally constraining the fit with a flux prediction. The sensitivity above $\Delta m_{41}^{2} \gtrsim 0.3 \mathrm{eV}^{2}$ would strongly improve but also become strongly model dependent. An interesting observation is the dip in sensitivity at $\Delta m_{41}^{2} \simeq 5 \times 10^{-2} \mathrm{eV}^{2}$. The effect is related to the interference of maximum and minimum disappearance for neutrinos from the two reactor cores to the two detectors, whose baselines differ by about $\sim 100 \mathrm{~m}$. A strong disappearance for signals of one of the reactor is counteracted by no disappearance for the other reactor. We have tested that the effect disappears when simulating the baseline of only one reactor core.

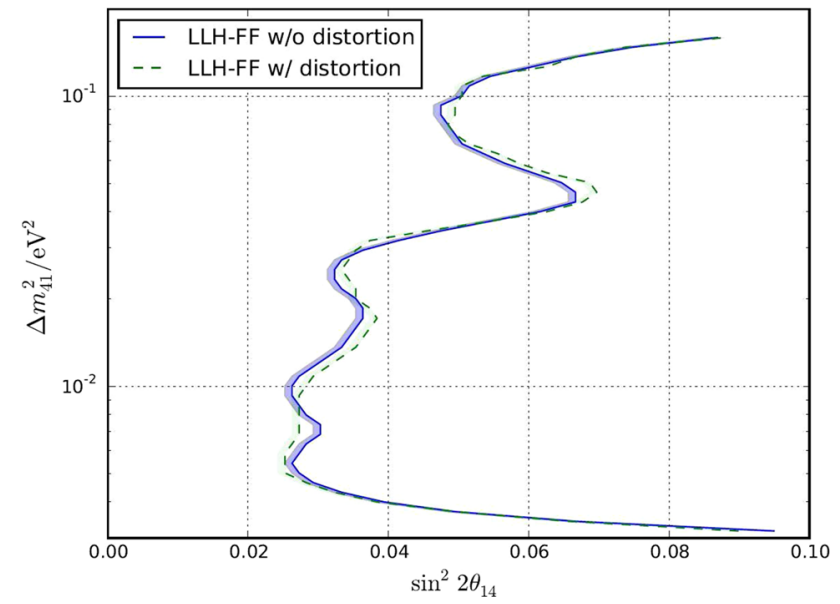

Fig. 8 Sensitivity (95\% C.L.) of the analysis as obtained from Asimov data sets with and without a spectral distortion at $5 \mathrm{MeV}$

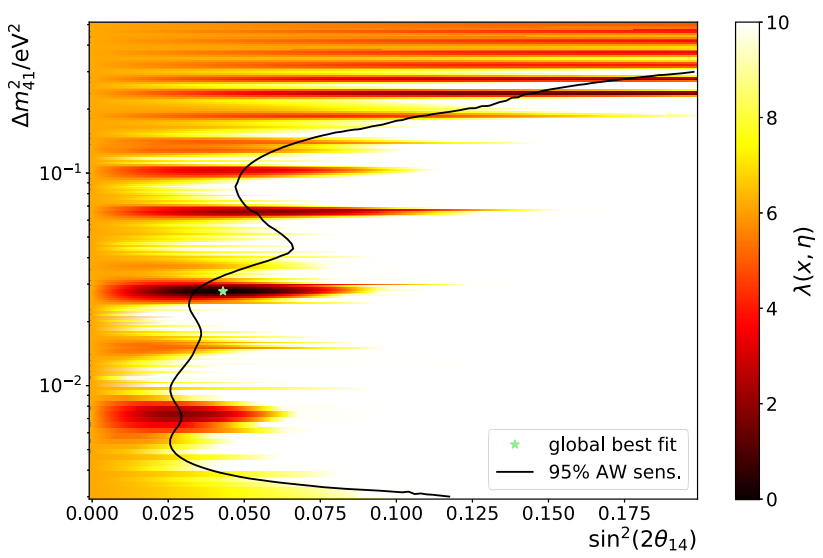

Fig. 9 Likelihood scan of the experimental data

The effect of the aforementioned spectral distortions of reactor flux models has been studied with two Asimov data sets. One of them included a bump-like distortion at $5 \mathrm{MeV}$ using a double-Gaussian approximation of the measurement in [23]. The resulting sensitivity is only marginally impacted as shown in Fig. 8.

\section{Experimental result}

The result of the scan of the test statistic $\lambda(\mathbf{x})$ for the experimental data is shown in Fig. 9.

The global best fit minimum is found for the values $\sin ^{2} \hat{2} \theta_{14}=0.043$ and $\Delta \hat{m}_{41}^{2}=0.028 \mathrm{eV}^{2}$. The nuisance parameters converged to values within their reasonable range. In particular the best fit value $\sin ^{2} 2 \theta_{13}=0.108_{-0.017}^{+0.016}$ of the null hypothesis is found in agreement with the nominal value $0.105+-0.014$ that has been obtained from the same data set [41]. The difference to that result is expected from the differences of the fit method and has been verified in 

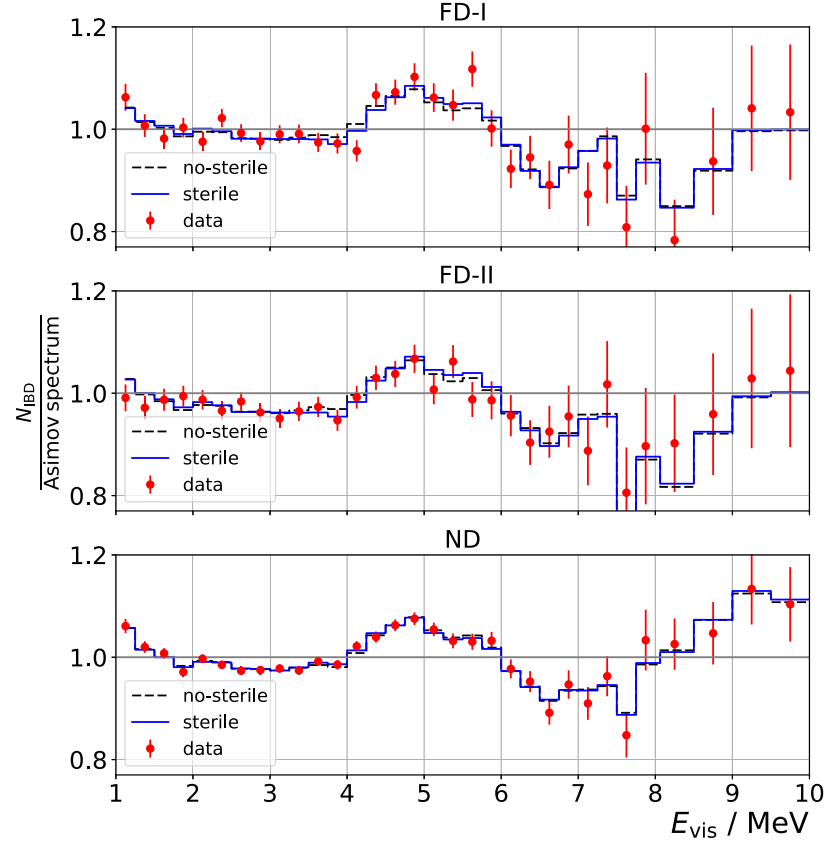

Fig. 10 Experimental residuals for the three consistently fitted data sets FD-I, FD-II, and ND. The data are normalized to the nominal reactor expectation [38] adapted to the Double Chooz reactors including conventional oscillations with parameters taken from an independent measurement [12]. The experimental data are plotted as red dots. The global best fit is shown as a solid line while for comparison the best-fit null hypothesis is shown as a dashed line. As the fit optimizes systematic uncertainties to the data, only statistical error bars are displayed

a detailed comparison of the fit methods. Also the value $\sin ^{2} 2 \theta_{13}=0.1077$ obtained for the global best fit $\hat{\eta}$ is very close to the null hypothesis and thus does not indicate a pull on the best fit.

The value of the test statistic of the best fit with respect to the null hypothesis of no sterile mixing is $\lambda\left(\mathbf{x}_{\text {exp }}\right)=6.15$. From 388 performed pseudo experiments of the null hypothesis in Fig. 6, a total of 96 have a larger or equal value of $\lambda$. The corresponding $\mathrm{p}$ value is $24.7 \pm 2.2 \%$. This $\mathrm{p}$ value does not depend on details of the modeling of the test statistic. When using the three approximations of the test statistic distributions in Fig. 6, very similar $p$ values between 22 to $26 \%$ are obtained. Therefore, the experimental result is fully consistent with the null hypothesis of no mixing with sterile neutrinos and no evidence for a signal can be reported.

The location of the best-fit point is not within the region of good sensitivity but close to the estimated sensitivity line, see Figs. 4 and 8. This is, as discussed above, an expected feature of statistical background fluctuations that are being picked up by a signal model.

Figure 10 shows the fit residuals normalized to the number of events expected for the nominal reactor-model including conventional oscillations. Also shown are the best fit of the null (non-sterile) and best-fit sterile hypothesis. All three data sets are consistently described by both models with a generally good agreement, including the observed bump at $5 \mathrm{MeV}$ and other spectral features, as expected from the implementation of the fit. No particular difference is observed between the three data sets that would hint to a mismodeled detector responses. Note that due to the use of a free but global normalization for each energy bin, the fit does not depend on the assumed shape and normalization of the initial reactor flux model but only on the consistency of the measured experimental data in the three data sets. The sterile model achieves a marginally better description. The difference can be quantified by Pearson's $\chi^{2}$-test [53]. The summed $\chi^{2}$ values of the three data distributions of Fig. 10 are 78.17 for the best-fit no-sterile model and 71.91 for the best-fit sterile model, respectively. With a rough estimation of the number of degrees of freedom of 76 , i.e. the number of data points corrected for the free overall normalizations of each energy bin, this indicates an acceptable goodness of fit for both models. The difference $\Delta \chi^{2}=6.25$ shows no systematic trend and is largely driven by a few fluctuating data points, i.e. the two energies 4.1 MeV and 5.6 MeV dominate the difference with a summed contribution of $\Delta \chi^{2}=5.5$. As discussed above, this is an expected behavior also for the no-sterile case where for each statistical fluctuation of data a matching sterile hypothesis can be constructed. No general trend in the data supporting a sterile signal is observed, which is consistent with the observation of an insignificant $p$ value as reported above.

\section{Discussion}

The experimental data has been tested over the full range of the two-dimensional signal parameter space. The globally found minimum does not constitute a significant observation of a signal but is well compatible with the null hypothesis of no mixing with sterile neutrinos.

In response to the limited computing resources that do not permit the evaluation of the test statistic with pseudo experiments at every point in the two-dimensional parameter space with sufficiently accurate coverage, we have decided for a more robust limit-setting strategy which is also known under the term raster-scan [45]. Here, we calculate onedimensional exclusion limits on the maximum allowed value of $\sin ^{2} 2 \theta_{14}$ as a function of $\Delta m_{41}^{2}$. These limits are calculated with a frequentist approach based on Wilks' theorem comparing the local test statistic with respect to the best fit at the probed $\Delta m_{41}^{2}$ and using the $\chi^{2}$ probability with one degree of freedom. The statistical coverage of the approach has been verified with pseudo experiments of injected signal as shown above.

Alternatively a two-dimensional approach could be pursued, where the test statistic is compared to the globally found 


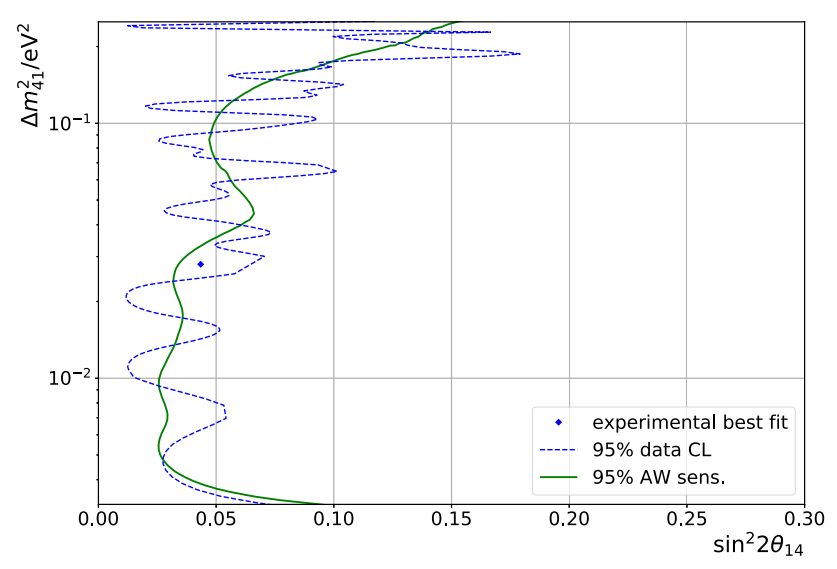

Fig. 11 Upper limit (dotted blue line) at 95\% C.L. for $\sin ^{2} 2 \theta_{14}$ as a function of $\Delta m_{41}^{2}$. The black dot indicates the position of the global best fit. The green solid line corresponds to the AW-sensitivity

maximum likelihood. Such a strategy has been followed e.g. for the analysis in Daya Bay [23]. Here the exclusion would correspond to the probability of the combination of $\sin ^{2} 2 \theta_{14}$ and $\Delta m_{41}^{2}$. However, pseudo experiments with an injected signal have revealed that our test statistic strongly depends on the injected value of $\sin ^{2} 2 \theta_{14}$. For small values of $\sin ^{2} 2 \theta_{14}$ it is close to the test statistic that we have observed for the null hypothesis (see Fig. 4) while it gradually crosses over into a $\chi^{2}$-distribution of two degrees of freedom for larger values. Because the determination of limits with correct statistical coverage would require the simulation of a very large number of pseudo experiments throughout the entire parameter space, we have chosen the raster-scan approach. Within the available computing resources this resulted in limits of more accurate coverage. We note that this strategy applies only to the setting of limits but not to the $p$ value of the analysis that has been obtained in a full two-dimensional approach.

The resulting exclusion limits are shown in Fig. 11. The obtained limits are generally close to the AW-sensitivity. For masses $\Delta m_{41}^{2}$, where the best fit results in the null hypothesis $\sin ^{2} 2 \theta_{14}=0$, the upper limit coincides with the median expected sensitivity. Due to statistical fluctuations in the data one expects variations around this median sensitivity, depending whether excesses or deficits in the prediction match these fluctuations better. As the allowed parameter space is bounded to positive values of $\sin ^{2} 2 \theta_{14}$, we expect roughly for $50 \%$ of probed $\Delta m_{41}^{2}$ values fits with a non-zero value of $\sin ^{2} 2 \theta_{14}$ resulting in less constraining limits than the average sensitivity and similarly a roughly equal number of more constraining limits.

As discussed above, both experiments Daya Bay and RENO probe a similar range of $L / E$ values and have published exclusion limits for a similar range of $\Delta m_{41}^{2}$ for sterile neutrino mixing in the $3+1$ model $[11,22,27]$. A comparison of these results is shown in Fig. 12. We note, that a

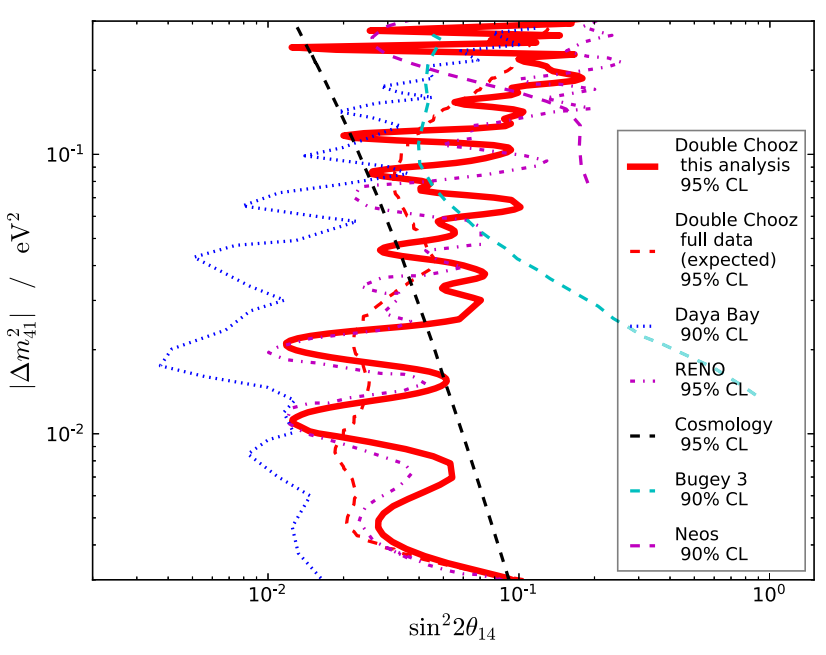

Fig. 12 Comparison of the upper exclusion limits provided by this analysis (Double Chooz) with results from other measurements: Daya Bay [11], RENO [27], Bugey-3 [32] Neos [42], and cosmological limits [10] based on the combination of observations of the cosmic microwave background, gravitational lensing and baryon-acoustic oscillations. Additionally displayed is the expected average sensitivity of Double Chooz with the full data statistics from the multi-detector phase. Note, that the figure combines 1-d and 2-d limits as well as limits of different confidence level

detailed quantitative comparison is difficult, because, unlike Double Chooz and as discussed above, the two other experiments provide two-dimensional limits. In order to quantify the difference, we have evaluated the consistency of the twodimensional and one-dimensional raster-scan approach with seven pseudo experiments of the null-hypothesis. It is found that the median line of the 2-d TS value of 8.7, corresponding to a $10 \%$ p value in Fig. 6 matches within about 10-20\% with the $90 \%$ AW-sensitivity. Furthermore, marginalizing the TS values of the 2-d contours of these pseudo experiments for every $\Delta m_{41}^{2}$ value and averaging the pseudo experiments matches well with the 1-d AW-sensitivity without noticeable bias. Another important difference with respect to the aforementioned experiments is that analysis assumptions differ. In particular, the Daya Bay result includes a reactor flux model and constraints on $\theta_{13}$. We have tested that such assumptions would also increase the sensitivity of this analysis. The statistics of $\bar{v}_{e}$ candidates used in Daya Bay and RENO is roughly four times the statistics used here. In addition, the figure shows limits obtained by the Bugey- 3 collaboration and limits from combining cosmological observations. The Double Chooz result based on the here used data is less constraining than Daya Bay but is competitive to the other presented results.

The result has been obtained under the assumption of a 3 +1 model. An extension to a $3+2$ model would require the extension of the $3 \times 3$ PMNS Matrix to 5 dimensions with 7 additional mixing angles plus additional CP phases and the oscillations would also involve additional mass differ- 
ences. In the simplest approximation, Eq. (1) would include an additional term $-\sin ^{2} 2 \theta_{15} \sin ^{2} \Delta m_{51}^{2} L /(4 E)$. This leads to additional oscillations, which potentially interfere with the 4-1 oscillation if $\Delta m_{41}^{2} \approx \Delta m_{51}^{2}$. As a result of test studies [37], we find that the here presented limits of the mixing angle as a function of $\Delta m^{2}$ are largely valid also for $3+2$ models with largely different mass difference and in particular if $\Delta m_{51}^{2} \gtrsim 0.3 e V^{2}$. In case both mass-square differences fall into the sensitive region of this analysis, the oscillation of the respective larger $\Delta m^{2}$ is largely washed out and results in a global normalization offset, to which the data-to-data fit of this analysis is insensitive. In summary, though different in statistical coverage, the test for a $3+1$ model is also sensitive for a signal of a more complicated model.

The relative impact of systematic uncertainties has been tested in terms of sensitivity for the null hypothesis and for relatively strong signals of $\sin ^{2} 2 \theta_{14}=0.1$ and varying values of $\Delta m_{41}^{2}$. It is found that the relative impact of systematic uncertainties on the total error increases towards smaller values $\Delta m_{41}^{2}$. E.g. for determining the value $\sin ^{2} 2 \theta_{14}=0.1$ the relative error changes from $\frac{\sigma_{\text {stat }}}{\sigma_{\text {tot }}}=99 \%$ for $\Delta m_{41}^{2}=0.1 \mathrm{eV}^{2}$ to $\frac{\sigma_{\text {stat }}}{\sigma_{\text {tot }}}=55 \%$ for $\Delta m_{41}^{2}=7.3 \times 10^{-3} \mathrm{eV}^{2}$. Among the different systematic parameters, the uncertainty of the energy scale and the unconstrained parameter $\theta_{13}$ show the largest impact on the total uncertainty. As the current analysis is limited by statistics, it will benefit from the full data set of Double Chooz. Figure 12 shows the expected median sensitivity for the full duration of multiple detector operation, corresponding to an increase in statistics by roughly a factor 2.4 In addition, we expect improvements by the off-reactor data set, that is enlarged from 7 to 32 days, resulting in reduced uncertainties in background modeling and furthermore the planned improved measurement of the proton number of the neutrino target.

\section{Summary}

We have presented an initial search for oscillations of electron anti-neutrinos with additional sterile neutrino flavors with the Double Chooz experiment. The search uses data from five years of operation of Double Chooz, including two years in the two-detector configuration. The analysis method is based on a profile likelihood, searching for the disappearance due to oscillations in a data-to-data comparison of the two respective detectors. The analysis is optimized for a $3+1$ model and is sensitive in the mass range $5 \times 10^{-2} \mathrm{eV}^{2} \lesssim \Delta m_{41}^{2} \lesssim 3 \times 10^{-1} \mathrm{eV}^{2}$. No significant disappearance signal additionally to the conventional oscillations related to $\theta_{13}$ is observed in a full 2-d scan of the model parameters. Correspondingly exclusion bounds on the sterile mixing parameter are determined in form of a raster-scan of $\theta_{14}$ as a function of $\Delta m_{41}^{2}$. The result is competitive to similar searches in this mass range. An update to the full data set from Double Chooz is planned.

Acknowledgements We thank the EDF ("Electricity of France") company; the European fund FEDER; the Région Grand Est (formerly known as the Région Champagne-Ardenne); the Département des Ardennes; and the Communauté de Communes Ardenne Rives de Meuse. We acknowledge the support of the CEA, CNRS/IN2P3, the computer centre CC-IN2P3 and LabEx UnivEarthS in France; the Max Planck Gesellschaft, the Deutsche Forschungsgemeinschaft DFG, the Transregional Collaborative Research Center TR27, the excellence cluster "Origin and Structure of the Universe" and the Maier-LeibnitzLaboratorium Garching in Germany; the Ministry of Education, Culture, Sports, Science and Technology of Japan (MEXT) and the Japan Society for the Promotion of Science (JSPS) in Japan; the Ministerio de Economía, Industria y Competitividad (SEIDI-MINECO) under grants FPA2016-77347-C2-1- P and MdM-2015-0509 in Spain; the Department of Energy and the National Science Foundation; the Russian Academy of Science, the Kurchatov Institute and the Russian Foundation for Basic Research (RFBR) in Russia; the Brazilian Ministry of Science, Technology and Innovation (MCTI), the Financiadora de Estudos e Projetos (FINEP), the Conselho Nacional de Desenvolvimento Científíco e Tecnológico (CNPq), the São Paulo Research Foundation (FAPESP) and the Brazilian Network for High Energy Physics (RENAFAE) in Brazil.

Data Availability Statement This manuscript has no associated data or the data will not be deposited. [Authors' comment: The analysis described in this manuscript is based on data that have been published in Ref. [4].

Open Access This article is licensed under a Creative Commons Attribution 4.0 International License, which permits use, sharing, adaptation, distribution and reproduction in any medium or format, as long as you give appropriate credit to the original author(s) and the source, provide a link to the Creative Commons licence, and indicate if changes were made. The images or other third party material in this article are included in the article's Creative Commons licence, unless indicated otherwise in a credit line to the material. If material is not included in the article's Creative Commons licence and your intended use is not permitted by statutory regulation or exceeds the permitted use, you will need to obtain permission directly from the copyright holder. To view a copy of this licence, visit http://creativecomm ons.org/licenses/by/4.0/.

Funded by $\mathrm{SCOAP}^{3}$.

\section{References}

1. K. Abazajian et al., Light sterile neutrinos: a white paper (2012). arXiv: 1204.5379

2. J.N. Abdurashitov et al., Measurement of the solar neutrino capture rate with gallium metal. III: results for the 2002-2007 data-taking period. Phys. Rev. C 80, 015807 (2009). https://doi.org/10.1103/ PhysRevC.80.015807. arXiv:0901.2200

3. Y. Abe et al., Indication for the disappearance of reactor electron antineutrinos in the Double Chooz experiment. Phys. Rev. Lett. 108, 131801 (2012a). https://doi.org/10.1103/PhysRevLett. 108.131801. arXiv:1112.6353

4. Y. Abe et al., Reactor electron antineutrino disappearance in the Double Chooz experiment. Phys. Rev. D 86, 052008 (2012b). https://doi.org/10.1103/PhysRevD.86.052008. arXiv:1207.6632 
5. Y. Abe et al., Direct measurement of backgrounds using reactor-off data in double Chooz. Phys. Rev. D 87, 011102 (2013). https://doi. org/10.1103/PhysRevD.87.011102. arXiv: 1210.3748

6. Y. Abe et al., Improved measurements of the neutrino mixing angle $\theta_{13}$ with the Double Chooz detector. JHEP 10, 086 (2014). https://doi.org/10.1007/JHEP02(2015)074. https:// doi.org/10.1007/JHEP10(2014)086. [Erratum: JHEP 02, 074 (2015)]. arXiv: 1406.7763

7. Y. Abe et al., Characterization of the spontaneous light emission of the PMTs used in the Double Chooz experiment. JINST 11(08), P08001 (2016). https://doi.org/10.1088/1748-0221/11/08/ P08001. arXiv:1604.06895

8. Y. Abreu, SoLid: an innovative anti-neutrino detector for searching oscillations at the SCK •CEN BR2 reactor. Nucl. Instrum. Methods A 845, 467-470 (2017). https://doi.org/10.1016/j.nima.2016.05. 119

9. M.A. Acero, C. Giunti, M. Laveder, Limits on nu(e) and anti-nu(e) disappearance from Gallium and reactor experiments. Phys. Rev. D 78, 073009 (2008). https://doi.org/10.1103/PhysRevD.78.073009. arXiv:0711.4222

10. M. Adams et al., Direct comparison of sterile neutrino constraints from cosmological data, $v_{e}$ disappearance data and $v_{\mu} \rightarrow v_{e}$ appearance data in a $3+1$ model (2020). arXiv:2002.07762

11. P. Adamson et al., Improved constraints on sterile neutrino mixing from disappearance searches in the MINOS, MINOS+, Daya Bay, and Bugey-3 experiments. Phys. Rev. Lett. 125(7), 071801 (2020). https://doi.org/10.1103/PhysRevLett.125.071801. arXiv:2002.00301

12. D. Adey et al., Measurement of the electron antineutrino oscillation with 1958 days of operation at Daya Bay. Phys. Rev. Lett. 121(24), 241805 (2018). https://doi.org/10.1103/PhysRevLett.121.241805. arXiv: 1809.02261

13. M. Agostini, B. Neumair, Statistical methods for the search of sterile neutrinos (2019). arXiv:1906.11854

14. A. Aguilar-Arevalo et al., Evidence for neutrino oscillations from the observation of anti-neutrino(electron) appearance in a antineutrino(muon) beam. Phys. Rev. D 64, 112007 (2001). https:// doi.org/10.1103/PhysRevD.64.112007. arXiv:hep-ex/0104049

15. A. Aguilar-Arevalo et al., Significant excess of electronlike events in the MiniBooNE Short-Baseline Neutrino experiment. Phys. Rev. Lett. 121(22), 221801 (2018). https://doi.org/10.1103/ PhysRevLett.121.221801. arXiv:1805.12028

16. Q.R. Ahmad et al., Direct evidence for neutrino flavor transformation from neutral current interactions in the Sudbury Neutrino Observatory. Phys. Rev. Lett. 89, 011301 (2002). https://doi.org/ 10.1103/PhysRevLett.89.011301. arXiv:nucl-ex/0204008

17. J. Ahn et al., Observation of reactor electron antineutrino disappearance in the RENO experiment. Phys. Rev. Lett. 108, 191802 (2012). https://doi.org/10.1103/PhysRevLett.108.191802. arXiv: 1204.0626

18. I. Alekseev et al., Search for sterile neutrinos at the DANSS experiment. Phys. Lett. B 787, 56-63 (2018). https://doi.org/10.1016/j. physletb.2018.10.038. arXiv:1804.04046

19. H. Almazán et al., Sterile neutrino constraints from the STEREO experiment with 66 days of reactor-on data. Phys. Rev. Lett. 121(16), 161801 (2018). https://doi.org/10.1103/PhysRevLett. 121.161801. arXiv: 1806.02096

20. H. Almazán Molina et al., Improved sterile neutrino constraints from the STEREO experiment with 179 days of reactor-on data (2019). arXiv: 1912.06582

21. F.P. An et al., Observation of electron-antineutrino disappearance at Daya Bay. Phys. Rev. Lett. 108, 171803 (2012). https://doi.org/ 10.1103/PhysRevLett.108.171803. arXiv:1203.1669

22. F.P. An et al., Improved search for a light sterile neutrino with the full configuration of the Daya Bay experiment. Phys. Rev. Lett.
117(15), 151802 (2016). https://doi.org/10.1103/PhysRevLett. 117.151802. arXiv: 1607.01174

23. F.P. An et al., Improved measurement of the reactor antineutrino flux and spectrum at Daya Bay. Chin. Phys. C 41(1), 013002 (2017). https://doi.org/10.1088/1674-1137/41/1/013002. arXiv: 1607.05378

24. M. Andriamirado et al., Note on arXiv:2005.05301. Preparation of the Neutrino-4 experiment on search for sterile neutrino and the obtained results of measurements (2020). arXiv:2006.13147

25. F. Ardellier et al., Double Chooz: a search for the neutrino mixing angle theta (13) (2006). arXiv:hep-ex/0606025

26. J. Ashenfelter et al., First search for short-baseline neutrino oscillations at HFIR with PROSPECT. Phys. Rev. Lett. 121(25), 251802 (2018). https://doi.org/10.1103/PhysRevLett.121.251802. 1806.02784

27. J. Choi et al., Search for sub-eV sterile neutrino at RENO (2020). arXiv:2006.07782

28. P. Coloma, P. Huber, T. Schwetz, Statistical interpretation of sterile neutrino oscillation searches at reactors. Eur. Phys. J. C 81(1), 2 (2021). https://doi.org/10.1140/epjc/ s10052-020-08774-2. arXiv:2008.06083

29. C.L. Cowan, F. Reines, F.B. Harrison, H.W. Kruse, A.D. McGuire, Detection of the free neutrino: a confirmation. Science 124, 103104 (1956). https://doi.org/10.1126/science.124.3212.103

30. G. Cowan, K. Cranmer, E. Gross, O. Vitells, Asymptotic formulae for likelihood-based tests of new physics. Eur. Phys. J. C 71, 1554 (2011). https://doi.org/10.1140/epjc/s10052-011-1554-0. https:// doi.org/10.1140/epjc/s10052-013-2501-z. [Erratum: Eur. Phys. J. C 73, 2501 (2013)]. arXiv:1007.1727

31. M. Danilov, Searches for sterile neutrinos at very short baseline reactor experiments. J. Phys. Conf. Ser. 1390(1), 012049 (2019). https://doi.org/10.1088/1742-6596/1390/1/012049. arXiv: 1812.04085

32. Y. Declais et al., Search for neutrino oscillations at 15-meters, 40meters, and 95-meters from a nuclear power reactor at Bugey. Nucl. Phys. B 434, 503-534 (1995). https://doi.org/10.1016/ 0550-3213(94)00513-E

33. M. Dentler, A. Hernández-Cabezudo, J. Kopp, P.A. Machado, M. Maltoni, I. Martinez-Soler, T. Schwetz, Updated global analysis of neutrino oscillations in the presence of $\mathrm{eV}$-scale sterile neutrinos. JHEP 08, 010 (2018). https://doi.org/10.1007/JHEP08(2018)010. arXiv:1803.10661

34. A. Esmaili, E. Kemp, O. Peres, Z. Tabrizi, Probing light sterile neutrinos in medium baseline reactor experiments. Phys. Rev. D 88, 073012 (2013). https://doi.org/10.1103/PhysRevD.88.073012. arXiv: 1308.6218

35. G.J. Feldman, R.D. Cousins, A unified approach to the classical statistical analysis of small signals. Phys. Rev. D 57, 3873-3889 (1998). https://doi.org/10.1103/PhysRevD.57.3873. arXiv:physics/9711021

36. Y. Fukuda et al., Evidence for oscillation of atmospheric neutrinos. Phys. Rev. Lett. 81, 1562-1567 (1998). https://doi.org/10.1103/ PhysRevLett.81.1562. arXiv:hep-ex/9807003

37. D. Hellwig, Search for light sterile neutrinos with the Double Chooz experiment. Dissertation, RWTH Aachen University, Aachen (2020). https://doi.org/10.18154/RWTH-2020-05375

38. P. Huber, On the determination of anti-neutrino spectra from nuclear reactors. Phys Rev C 84, 024617 (2011). https://doi.org/ 10.1103/PhysRevC.85.029901. [Erratum: Phys. Rev. C 85, 029901 (2012)]. arXiv:1106.0687

39. F. Kaether, W. Hampel, G. Heusser, J. Kiko, T. Kirsten, Reanalysis of the GALLEX solar neutrino flux and source experiments. Phys. Lett. B 685, 47-54 (2010). https://doi.org/10.1016/j. physletb.2010.01.030. arXiv:1001.2731

40. H. de Kerret et al., Yields and production rates of cosmogenic ${ }^{9} \mathrm{Li}$ and ${ }^{8} \mathrm{He}$ measured with the Double Chooz near and far detectors. 
JHEP 11, 053 (2018). https://doi.org/10.1007/JHEP11(2018)053. arXiv: 1802.08048

41. H. de Kerret et al., First Double Chooz $\theta_{13}$ measurement via total neutron capture detection. Nat. Phys. 16 (2020). https://doi.org/10. 1038/s41567-020-0831-y. arXiv:1901.09445

42. Y.J. Ko et al., Sterile neutrino search at the NEOS Experiment. Phys. Rev. Lett. 118(12), 121802 (2017). https://doi.org/10.1103/ PhysRevLett.118.121802. arXiv: 1610.05134

43. J. Kopp, P.A.N. Machado, M. Maltoni, T. Schwetz, Sterile neutrino oscillations: the global picture. JHEP 05, 050 (2013). https://doi. org/10.1007/JHEP05(2013)050. arXiv:1303.3011

44. E. Kwon, Precise measurement of reactor antineutrino spectrum flux and spectrum at RENO. J. Phys. Conf. Ser. 888(1), 012137 (2017). https://doi.org/10.1088/1742-6596/888/1/012137

45. L. Lyons, Raster scan or 2-D approach? (2014) arXiv:1404.7395

46. Z. Maki, M. Nakagawa, S. Sakata, Remarks on the unified model of elementary particles. Prog. Theor. Phys. 28, 870-880 (1962). https://doi.org/10.1143/PTP.28.870. [34 (1962)]

47. G. Mention, M. Fechner, T. Lasserre, T.A. Mueller, D. Lhuillier, M. Cribier, A. Letourneau, The reactor antineutrino anomaly. Phys. Rev. D 83, 073006 (2011). https://doi.org/10.1103/PhysRevD.83. 073006. arXiv: 1101.2755

48. C. Patrignani et al., Review of particle physics. Chin. Phys. C 40(10), 100001 (2016). https://doi.org/10.1088/1674-1137/40/10/ 100001

49. B. Pontecorvo, Neutrino experiments and the problem of conservation of leptonic charge. Sov. Phys. JETP 26, 984-988 (1968). [Zh. Eksp. Teor. Fiz. 53, 1717 (1967)]
50. S. Schoppmann, First measurement of $\theta_{13}$ with the final two detector setup of the Double Chooz. PhD thesis, RWTH Aachen University (2016). https://doi.org/10.18154/RWTH-2016-11063

51. A. Serebrov, R. Samoilov, A comment on the note arXiv:2006.13147 on arXiv:2005.05301. Preparation of the Neutrino-4 experiment on search for sterile neutrino and the obtained results of measurements (2020). arXiv:2006.13639

52. A.P. Serebrov et al., First observation of the oscillation effect in the Neutrino-4 experiment on the search for the sterile neutrino. Pisma Zh Eksp Teor Fiz 109(4), 209-218 (2019). https://doi.org/ 10.1134/S0021364019040040. [JETP Lett. 109(4), 213 (2019)]. arXiv: 1809.10561

53. M. Tanabashi et al., Review of particle physics. Phys. Rev. D 98(3), 030001 (2018). https://doi.org/10.1103/PhysRevD.98.030001

54. P. Vogel, J.F. Beacom, Angular distribution of neutron inverse beta decay, anti-neutrino(e). Phys. Rev. D 60, 053003 (1999). https:// doi.org/10.1103/PhysRevD.60.053003. arXiv:hep-ph/9903554

55. M. Wallraff, C. Wiebusch, Calculation of oscillation probabilities of atmospheric neutrinos using nuCraft. Comput. Phys. Commun. 197, 185-189 (2015). https://doi.org/10.1016/j.cpc.2015.07.010. arXiv: 1409.1387

56. S.S. Wilks, The large-sample distribution of the likelihood ratio for testing composite hypotheses. Ann. Math. Stat. 9(1), 60-62 (1938). https://doi.org/10.1214/aoms/1177732360 